IUCrJ

ISSN 2052-2525

MATERIALS|COMPUTATION

Received 21 October 2019

Accepted 21 November 2019

Edited by A. Fitch, ESRF, France

Keywords: thermoelectric materials; zinc antimonides; pair distribution function; operando study; powder X-ray diffraction; electrical resistance.

Supporting information: this article has supporting information at www.iucrj.org

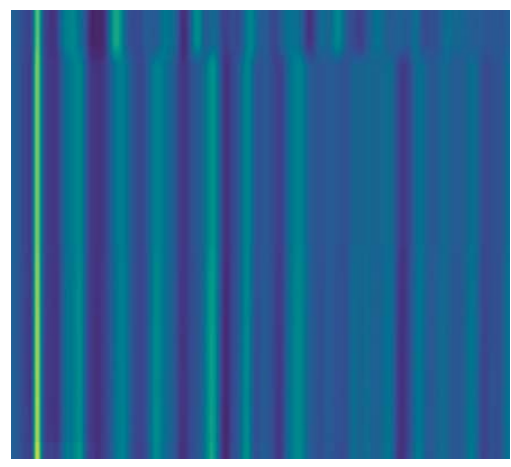

\section{Operando X-ray scattering study of thermoelectric $\beta-\mathrm{Zn}_{4} \mathrm{Sb}_{3}$}

\author{
Lasse Rabøl Jørgensen, Christian Moeslund Zeuthen, Kasper Andersen Borup, \\ Martin Roelsgaard, Nils Lau Nyborg Broge, Jonas Beyer and Bo Brummerstedt \\ Iversen*
}

Department of Chemistry, University of Aarhus, Langelandsgade 140, Aarhus C 8000, Denmark. *Correspondence e-mail: bo@chem.au.dk

The application of thermoelectrics for energy harvesting depends strongly on operational reliability and it is therefore desirable to investigate the structural integrity of materials under operating conditions. We have developed an operando setup capable of simultaneously measuring X-ray scattering data and electrical resistance on pellets subjected to electrical current. Here, operando investigations of $\beta-\mathrm{Zn}_{4} \mathrm{Sb}_{3}$ are reported at current densities of $0.5,1.14$ and $2.3 \mathrm{~A} \mathrm{~mm}^{-2}$. At $0.5 \mathrm{~A} \mathrm{~mm}^{-2}$ no sample decomposition is observed, but Rietveld refinements reveal increased zinc occupancy from the anode to the cathode demonstrating zinc migration under applied current. At $1.14 \mathrm{~A} \mathrm{~mm}^{-2} \beta-\mathrm{Zn}_{4} \mathrm{Sb}_{3}$ decomposes into $\mathrm{ZnSb}$, but pair distribution function analysis shows that $\mathrm{Zn}_{2} \mathrm{Sb}_{2}$ units are preserved during the decomposition. This identifies the mobile zinc in $\beta-\mathrm{Zn}_{4} \mathrm{Sb}_{3}$ as the linkers between the $\mathrm{Zn}_{2} \mathrm{Sb}_{2}$ units. At $2.3 \mathrm{~A} \mathrm{~mm}^{-2}$ severe Joule heating triggers transition into the $\gamma-\mathrm{Zn}_{4} \mathrm{Sb}_{3}$ phase, which eventually decomposes into $\mathrm{ZnSb}$, demonstrating $\mathrm{Zn}$ ion mobility also in $\gamma-\mathrm{Zn}_{4} \mathrm{Sb}_{3}$ under electrical current.

\section{Introduction}

Thermoelectric (TE) materials are capable of interconverting thermal and electrical energy and they provide exciting opportunities for green harvesting of the vast amounts of waste heat released e.g. in engines or industrial processes. Many TE materials are explored for power-generation applications, such as silicides (Sadia et al., 2016), PbTe (Pei et al., 2011), clathrates (Johnsen et al., 2006), antimonides (Pedersen et al., 2007) and half-Heuslers (Appel \& Gelbstein, 2014). Apart from the obvious need for high performance, low cost and environmentally friendly materials, one of the main obstacles of TE technology is the lack of material stability during operation at high temperature with the material subjected to large temperature gradients and electrical current. Material degradation has been encountered not only in recent high-performance materials such as $\mathrm{SnSe}, \mathrm{Cu}_{2} \mathrm{Se}$ and $\mathrm{Zn}_{4} \mathrm{Sb}_{3}$ (Li et al., 2016; Brown et al., 2014; Hung et al., 2017; Yin et al., 2010), but even in inorganic clathrates usually considered as extremely stable compounds (Reardon et al., 2017). TE energy harvesting relies strongly on optimizing operational reliability without compromising energy-conversion efficiency.

Studies of the stability of TE materials typically use experimental conditions that do not resemble an operating module. This primarily involves powder X-ray diffraction (PXRD) studies at elevated temperatures, or thermal cycling, using various atmospheres (Jørgensen et al., 2018a, 2018b; Pedersen \& Iversen, 2008; Pedersen et al., 2006, 2010; Fischer 
et al., 2018; Reardon et al., 2017; Yin et al., 2010). Some studies have exposed TE materials to (semi)realistic conditions but they have not simultaneously characterized the structural or TE properties (operando) (Reardon et al., 2017; Hung et al., 2017; Yin et al., 2014). Operando studies are well established e.g. for battery materials (Senyshyn et al., 2012; Ulvestad et al., 2015; Taminato et al., 2016) or catalysts (Topsøe, 2003). Indeed, operando characterization is invaluable to battery research, where atomistic understanding of ion diffusion and structural stability during (dis)charging cycles is essential for improving battery technology (Nelson et al., 2012; Cuisinier et al., 2013). Here we introduce an experimental setup capable of mimicking real-life TE conditions. By using high-energy synchrotron radiation $(60 \mathrm{keV})$ to penetrate samples it allows characterization of both atomic structure and electrical resistance of densified bulk samples exposed to direct current.

The effect of electrical current on TE materials is rarely investigated in stability studies but it is particularly important for mixed ionic electronic conductors such as $\mathrm{Cu}_{2} \mathrm{Se}$ and $\beta$ $\mathrm{Zn}_{4} \mathrm{Sb}_{3}$. In these materials, the coexistence of a rigid anion crystal structure and a liquid-like cation sublattice causes the materials to possess a low thermal conductivity and a relatively high electrical conductivity making them highly suitable as TE materials (Caillat et al., 1997). Conversely, having highly mobile ions in the structure may not be ideal for reliable longterm operation in electric current. This has been the essential challenge for $\beta-\mathrm{Zn}_{4} \mathrm{Sb}_{3}$, which has $\mathrm{Zn}$ migrating towards the cathode end of the material, that is, in the direction of the current during spark plasma sintering (SPS) (Yin et al., 2014, 2012). Since only ex situ characterization methods have been employed in studying the $\mathrm{Zn}$ migration, the atomistic insight into the process is limited. Here we report an operando study of densified rod-shaped samples of $\beta-\mathrm{Zn}_{4} \mathrm{Sb}_{3}$ subjected to electrical current, and three successful high-energy synchrotron X-ray data collections were achieved. For two current settings the detector was placed to provide PXRD data, while for the third current setting, total scattering data were obtained at short detector distances allowing for subsequent pair distribution function (PDF) analysis.

$\beta-\mathrm{Zn}_{4} \mathrm{Sb}_{3}$ crystallizes in space group $R \overline{3} c$ with $\mathrm{Zn}$ at the $36 f$ site (denoted $\mathrm{Zn} 1$ ), and $\mathrm{Sb}$ at the $18 e$ and $12 c$ sites (denoted $\mathrm{Sb} 1$ and Sb2, respectively), Fig. 1(a). The space- and timeaveraged crystal structure has three interstitial $\mathrm{Zn}$ sites in close proximity to the main $\mathrm{Zn} 1$ site [not included in Fig. 1(a)] (Snyder et al., 2004). The refined stoichiometry of $\mathrm{Zn}_{3.83(4)} \mathrm{Sb}_{3}$ is consistent with the observed mass density, which was not the case for earlier models (Mayer et al., 1978). However, later studies have revealed a certain compositional freedom in the Zn content (Toberer et al., 2010). Here we refer to the compound as $\beta-\mathrm{Zn}_{4} \mathrm{Sb}_{3}$ noting that this does not reflect the true composition.

\section{Experimental}

The experiments were carried out at beamline P02.1 at PETRA III, DESY, Germany. Because of the high photon energy $(60 \mathrm{keV})$, it is possible to penetrate a densified inorganic sample of $1 \mathrm{~mm}$ thickness without extensive absorption ( $\mu R<1.4$ for $\beta-\mathrm{Zn}_{4} \mathrm{Sb}_{3}$, assuming crystallographic density, with $\mu$ being the linear absorption coefficient and $R$ half the sample thickness). The samples were subjected to direct current by each sample being spring loaded between two electrodes, Fig. 2(a). The current was kept constant during the $\mathrm{X}$-ray experiment. Two molybdenum wires were placed in contact with the sample surface to measure the four point resistance between the two points of contact during the experiment. When measuring total scattering data, a sampleto-detector distance of $\sim 200 \mathrm{~mm}$ was used with the detector placed such that the beam center was at the edge of the detector. This resulted in a maximum $Q$ value of $\sim 33 \AA^{-1}$. For PXRD, the sample-to-detector distance was $\sim 650 \mathrm{~mm}$ to improve angular resolution and the beam center was moved to the center of the detector to increase the detected intensity. The sample-to-detector distances and the instrumental resolution were calibrated using a $\mathrm{LaB}_{6}$ NIST sample. The resulting scattering patterns were azimuthally integrated using the Dioptas software (Prescher \& Prakapenka, 2015) and the TS data was Fourier transformed in PDFGet $X 3$ to obtain the PDF (Juhás et al., 2013). Refinement of PXRD data was carried out using the FullProf (Rodríguez-Carvajal, 1993) and JANA2006 (Petrícek et al., 2014) softwares, and the PDF data 


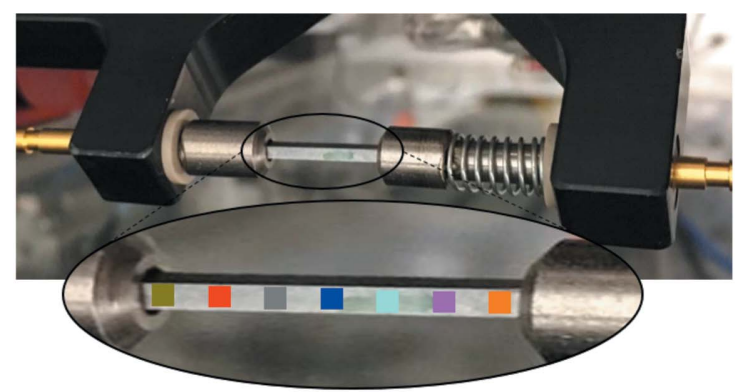

(a)

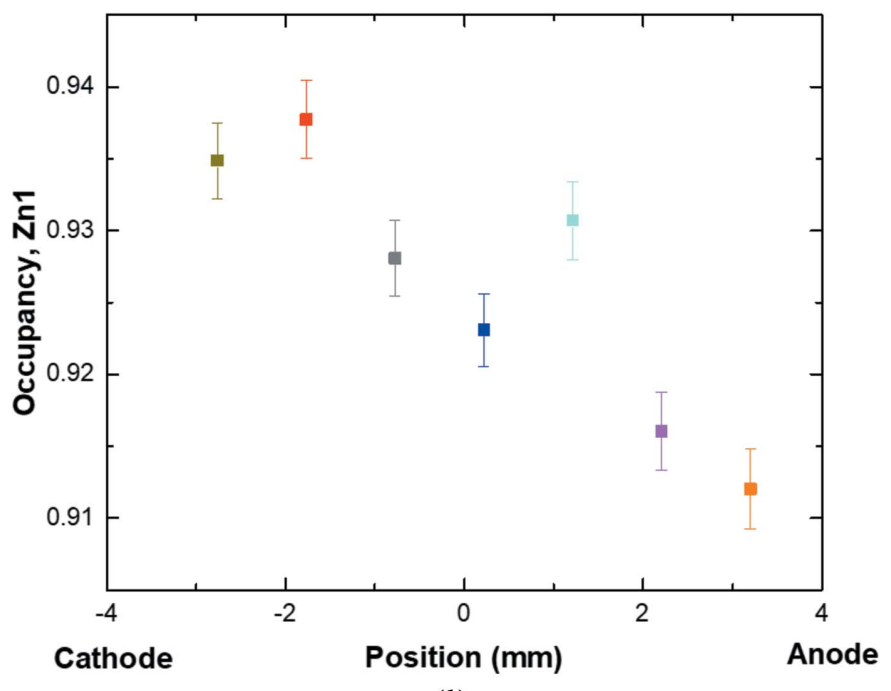

(b)

Figure 2

(a) The experimental setup installed at beamline P02.1, where the sample is spring loaded between two electrodes and placed in front of the X-ray beam. (b) Refined occupancy parameters for the $\mathrm{Zn} 1$ site at different positions indicated by the colored squares in $(a)$.

was refined using PDFgui (Farrow et al., 2007). A detailed description of the refinement models is given in the Supporting information.

The synthesis of $\beta-\mathrm{Zn}_{4} \mathrm{Sb}_{3}$ was carried out using the method of Yin et al. (2014). Zinc powder (99.99\%, Alfa Aesar) and antimony powder $(99.5 \%$, ChemPur) were weighed in a stoichiometric ratio $\left(\mathrm{Zn}_{4} \mathrm{Sb}_{3}\right)$ and mixed for 15 mins in a ball mill (SpectroMill, Chemplex Industries, Inc.). The powder was then transferred to a graphite die with a 1 inch diameter and compacted using SPS. To compensate for zinc migration caused by the current in the SPS press, a zinc foil $(99.95 \%$, Sterilin) of $0.15 \mathrm{~mm}$ thickness was placed at the anode of the pellet, resulting in a compositionally homogenous sample as verified by PXRD.

\section{Results}

The present experiments used $60 \mathrm{keV}$ synchrotron radiation and current densities of $0.5,1.14$ and $2.3 \mathrm{~A} \mathrm{~mm}^{-2}$. These current densities are considerably higher than for operating TE modules but this was necessary because of the limited beam time, and it provides an accelerated stability test. The current densities are similar in magnitude to typical SPS densification processes. The first experiment used $0.5 \mathrm{~A} \mathrm{~mm}^{-2}$ and PXRD data were recorded, see Fig. S1 in the Supporting information. During 270 mins no structural transitions were observed. Time-resolved sequential Rietveld refinements were carried out to track changes in unit-cell parameters and atomic displacement parameters (ADPs), and the refined parameters are shown in Fig. S2. Joule heating of the sample causes a rapid increase in both unit-cell parameters and ADPs corresponding to a temperature of $\sim 65^{\circ} \mathrm{C}$ immediately after initiating the current exposure. This sample temperature was estimated using the lattice expansion coefficient obtained from a separate variable-temperature PXRD experiment conducted at beamline I15-1 at the Diamond Light Source (Fig. S3). The expansion was found to be $2.15 \times 10^{-4} \AA \mathrm{K}^{-1}$ and $2.37 \times 10^{-4} \AA_{\mathrm{A} \mathrm{K}}^{-1}$ for the $a$ axis and $c$ axis, respectively. Since the measured sample electrical resistance does not at any time exceed the value measured right after initiating the current (Fig. S1), the sample temperature must reach steady state, and the subsequent relative development of the unit-cell parameters and ADPs is primarily because of structural changes (Zn migration). Similar behaviors are observed for the ADPs of all three atomic sites (Fig. S2), with a linear increase during the entire experiment. The absolute changes in ADPs are largest for the $\mathrm{Zn}$ sites. Decreasing the $\mathrm{Zn}$ content on the $\mathrm{Zn}$ sites, because of migration, will lead to lower average electron density on those sites. Since the present Rietveld model does not vary the site occupancy factor, the effect will be reflected in an increased ADP.

The sample was expected to form a $\mathrm{Zn}$ concentration gradient and this was confirmed from refinement of PXRD data collected along the sample with a spatial resolution of $1 \mathrm{~mm}$ and sufficient data quality to extract the site occupancy factor for the $\mathrm{Zn}$ sites. The obtained occupancy for the $\mathrm{Zn} 1$ site is shown in Fig. 2(b), whereas the occupancies of the interstitial Zn sites (Zn2, Zn3, Zn4) are shown in Fig. S4(b). The overall composition of the structure is plotted in Fig. S4(c). Indeed, the occupancy of the $\mathrm{Zn} 1$ site increases when moving from the anode towards the cathode, but this behavior is not seen for the interstitial $\mathrm{Zn}$ sites, where the occupancy is approximately constant throughout the sample. The importance of the interstitial sites in the $\mathrm{Zn}$ migration process has been suggested but never quantified (Dasgupta et al., 2013). At first sight, the disordered low-occupancy $\mathrm{Zn}$ interstitial sites may be expected to behave somewhat like a liquid, but it appears that it is the main $\mathrm{Zn} 1$ that provides ion migration during current exposure. The total $\mathrm{Zn}$ content increases from the anode to the cathode, although the absolute values may be inaccurate since the ADPs are fixed to literature values (ICSD No. 159090; Pedersen et al., 2007). Even so, the relative values are expected to be reliable.

Next, we analyze the PDF data measured with a current density of $1.14 \mathrm{~A} \mathrm{~mm}^{-2}$, Fig. 3(b). After $~ 55$ mins, an abrupt decomposition of $\beta-\mathrm{Zn}_{4} \mathrm{Sb}_{3}$ into $\mathrm{ZnSb}$ is observed. Even though many of the characteristic distances within the structure change during the decomposition, the shortest distances at $r \simeq 2.7-2.9 \AA$ are maintained throughout the experiment, 
indicating that a substructure of $\beta-\mathrm{Zn}_{4} \mathrm{Sb}_{3}$ is preserved in $\mathrm{ZnSb}$. The $r \simeq 2.7-2.9 \AA$ correlations originate from rhomboid $\mathrm{Zn}_{2} \mathrm{Sb}_{2}$ units (Fig. 1) and this motif is present in both $\beta-\mathrm{Zn}_{4} \mathrm{Sb}_{3}$ and $\mathrm{ZnSb}$ with the only difference being the linking of different units by additional $\mathrm{Zn}$ in $\mathrm{Zn}_{4} \mathrm{Sb}_{3}$ (absent in $\mathrm{ZnSb}$ ). The rhomboid unit has been shown to be preserved through the phase transition from the low-temperature $\alpha-\mathrm{Zn}_{4} \mathrm{Sb}_{3}$ structure to the $\beta-\mathrm{Zn}_{4} \mathrm{Sb}_{3}$ structure (Nylén et al., 2007). Observing the same unit in $\mathrm{ZnSb}$ indicates that the rhomboid $\mathrm{Zn}_{2} \mathrm{Sb}_{2}$ substructure could be a fundamental feature of zinc antimonide structures. To confirm that the correlations at $r \simeq$ 2.7-2.9 $\AA$ originate from the rhomboid unit, PDFs have been simulated for the $\mathrm{Zn}_{2} \mathrm{Sb}_{2}$ units present in $\beta-\mathrm{Zn}_{4} \mathrm{Sb}_{3}$ and $\mathrm{ZnSb}$, and compared with the full PDF of $\beta-\mathrm{Zn}_{4} \mathrm{Sb}_{3}$ and $\mathrm{ZnSb}$, Fig. S7. The rhomboid unit is clearly the main contributor to this correlation.

The integrated intensity of the rhomboid PDF peak as a function of time reveals three distinct regions, Fig. 3(c). Up to $\sim 30$ mins, the intensity decreases only slightly, but in the following 20 mins there is a pronounced decrease. After 50 mins the intensity drops drastically, immediately before the decomposition of $\beta-\mathrm{Zn}_{4} \mathrm{Sb}_{3}$ at $\sim 55$ mins. A decrease in PDF intensity indicates less correlations at this distance, as expected because of the linking of the rhomboids in $\beta-\mathrm{Zn}_{4} \mathrm{Sb}_{3}$, which is not present in $\mathrm{ZnSb}$ (see Fig. 1). This is consistent with the refined occupancies in Fig. 2(b), where the $\mathrm{Zn} 1$ site becomes depleted because of migration. The present operando data suggest that it is the linking $\mathrm{Zn} 1$ that leave the $\beta-\mathrm{Zn}_{4} \mathrm{Sb}_{3}$ structure during decomposition to $\mathrm{ZnSb}$. It is presumably these $\mathrm{Zn}$ atoms that migrate during exposure to electrical current leading to a change in the $\mathrm{Zn} 1$ occupancy. The difference in slope in the three regions of Fig. 3(c) can be explained by the continuously changing composition, which is known to significantly change the diffusion constant of $\mathrm{Zn}$ in $\beta-\mathrm{Zn}_{4} \mathrm{Sb}_{3}$ (Løvvik et al., 2011). The electrical resistance is relatively constant during the first 45 mins after which it increases drastically. This increase indicates that the decomposition has occurred at the position of the first resistance probe, which is mounted $\sim 1 \mathrm{~mm}$ from the X-ray beam position. Thus, the decomposition commences in the end of the

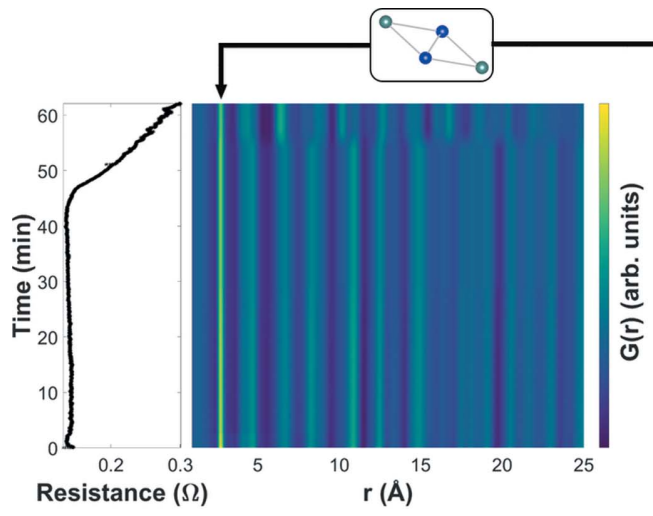

(a) (b)

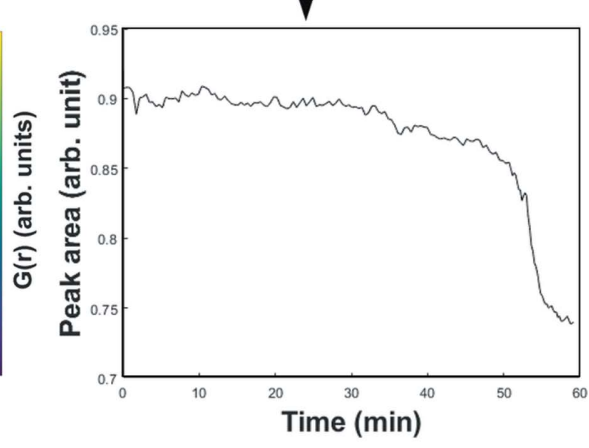

(c)
Figure 3

(a) Electrical resistivity and (b) surface plot of the raw PDF data. (c) A single-peak integration of the peak at $r \simeq 2.7 \AA$ seen in $(b)$. sample where the current enters, and progresses along the current direction. The $\mathrm{ZnSb}$ phase has a higher electrical resistivity compared with that of $\beta-\mathrm{Zn}_{4} \mathrm{Sb}_{3}$ and will therefore be heated more by the current (Shaver \& Blair, 1966; Caillat et al., 1997). The continuously increasing resistance reflects that a larger portion of the sample between the two resistance probes decomposes into $\mathrm{ZnSb}$. Calculating the average temperature of the sample between the two probes from the change in electrical resistance would thus not be representative of the true complex temperature landscape of the sample. Furthermore, the average temperature between the resistance probes (a distance of $8-10 \mathrm{~mm}$ ) is difficult to relate to the section illuminated by the $\mathrm{X}$-rays $(1 \times 1 \mathrm{~mm})$.

Real-space PDF refinements were performed to quantify the structural behavior leading up to the decomposition, and the corresponding unit-cell parameters and ADPs are shown in Fig. S5. From the sudden increase in the refined unit-cell parameters, a temperature increase of $145^{\circ} \mathrm{C}$ is estimated from Joule heating, which remains constant until the decomposition, as seen in the electrical resistance of the sample, Fig. 3(a). The subsequent behavior of the unit-cell parameters and ADPs correspond well to those obtained at $0.5 \mathrm{~A} \mathrm{~mm}^{-2}$ (a linear increase after initial Joule heating). This linear region extends to $\sim 40$ mins, where electrical contact resistance in the interface between the $\beta-\mathrm{Zn}_{4} \mathrm{Sb}_{3}$ and $\mathrm{ZnSb}$ phases causes further heating in this specific region of the sample.

In the last experiment at $2.3 \mathrm{~A} \mathrm{~mm}^{-2}$, PXRD data were collected, Fig. S6. $\beta-\mathrm{Zn}_{4} \mathrm{Sb}_{3}$ is present in the very beginning but a significant peak shift towards lower angles indicates that severe Joule heating increases the sample temperature rapidly. The Joule heating is proportional to the square of the applied current, i.e. a fourfold and 16 -fold increase compared with the first and second experiment (assuming equal absolute resistivity). Within seconds, a phase transition is seen from $\beta$ $\mathrm{Zn}_{4} \mathrm{Sb}_{3}$ to the high-temperature $\gamma-\mathrm{Zn}_{4} \mathrm{Sb}_{3}$ phase, which is stable above $493^{\circ} \mathrm{C}$ (Mozharivskyj et al., 2004). This phase is present for two minutes before it decomposes into $\mathrm{ZnSb}$ because of migration of $\mathrm{Zn}$ out of the structure. This means that $\mathrm{Zn}$ is also mobile in $\gamma-\mathrm{Zn}_{4} \mathrm{Sb}_{3}$, but additional studies are needed to establish the migration mechanism for this transition. Nevertheless, simulation of the PDF of $\gamma-\mathrm{Zn}_{4} \mathrm{Sb}_{3}$ reveals the same correlation at $r \simeq 2.7-2.9 \AA$ as seen in both $\beta-\mathrm{Zn}_{4} \mathrm{Sb}_{3}$ and $\mathrm{ZnSb}$ (Fig. S7).

This high-current experiment emphasizes the difficulty of separating current and temperature effects because of the lack of independent control. Experimental measurement of the sample temperature is challenging since placing a thermocouple at the sample surface would give a coldfinger effect, and infrared cameras are imprecise. Independent control of temperature and current would enable a more detailed analysis of the origin of decomposition and we will attempt 
to improve this aspect in the next generation of the operando setup.

\section{Conclusions}

In summary, the present study introduces operando measurements on TE materials by simultaneous collection of high-energy $\mathrm{X}$-ray scattering and electrical resistance data on dense pellets subjected to electrical current. Three $\beta-\mathrm{Zn}_{4} \mathrm{Sb}_{3}$ samples were exposed to current densities of $0.5,1.14$ and $2.3 \mathrm{~A} \mathrm{~mm}^{-2}$, and both ion migration and decomposition reactions were quantified directly based on analysis of PXRD and PDF data. The operando setup also holds potential for studies of solid-state battery electrolytes or piezo and ferroelectric materials, for example.

\section{Acknowledgements}

We gratefully acknowledge the beam time obtained at beamline P02.1 at DESY and at beamline I15-1 at the Diamond Light Source. We thank Robin Lefevré and Michael Wharmby for help during the beam time at DESY. Affiliation with the Aarhus University Center for Integrated Materials Research (iMAT) is gratefully acknowledged.

\section{Funding information}

This work was supported by the Danish National Research Foundation (Center for Materials Crystallography, DNRF93).

\section{References}

Appel, O. \& Gelbstein, Y. (2014). J. Electron. Mater. 43, 1976-1982. Brown, D. R., Day, T., Caillat, T. \& Snyder, G. J. (2014). J. Electron. Mater. 42, 2014-2019.

Caillat, T., Fleurial, J.-P. \& Borshchevsky, A. (1997). J. Phys. Chem. Solids, 58, 1119-1125.

Cuisinier, M., Cabelguen, P. E., Evers, S., He, G., Kolbeck, M., Garsuch, A., Bolin, T., Balasubramanian, M. \& Nazar, L. F. (2013). J. Phys. Chem. Lett. 4, 3227-3232.

Dasgupta, T., Stiewe, C., Sesselmann, A., Yin, H., Iversen, B. B. \& Mueller, E. (2013). J. Appl. Phys. 113, 103708.

Juhás, P., Davis, T., Farrow, C. L. \& Billinge, S. J. L. (2013). J. Appl. Cryst. 46, 560-566.

Farrow, C. L., Juhas, P., Liu, J. W., Bryndin, D., Božin, E. S., Bloch, J., Proffen, T. \& Billinge, S. J. L. (2007). J. Phys. Condens. Matter, 19, 335219.

Fischer, K. F. F., Jørgensen, L. R., Reardon, H., Zhang, J. \& Iversen, B. B. (2018). Phys. Chem. Chem. Phys. 20, 9930-9937.

Hung, L. T., Ngo, D. T., Han, L., Iversen, B. B., Yin, H., Pryds, N. \& Van Nong, N. (2017). Adv. Electron. Mater. 3, 1-8.

Johnsen, S., Bentien, A., Madsen, G. K. H., Iversen, B. B. \& Nygren, M. (2006). Chem. Mater. 18, 4633-4642.

Jørgensen, L. R., Zeuthen, C. B., Reardon, H. \& Iversen, B. B. (2018a). J. Phys. Chem. C, 122, 5317-5324.
Jørgensen, L. R., Zhang, J., Zeuthen, C. B. \& Iversen, B. B. (2018b). J. Mater. Chem. A, 6, 17171-17176.

Li, Y., He, B., Heremans, J. P. \& Zhao, J.-C. (2016). J. Alloys Compd. 669, 224-231.

Løvvik, O. M., Rauwel, P. \& Prytz, Ø. (2011). Comput. Mater. Sci. 50, 2663-2665.

Mayer, H. W., Mikhail, I. \& Schubert, K. (1978). J. Less-Common Met. 59, 43-52.

Momma, K. \& Izumi, F. (2011). J. Appl. Cryst. 44, 1272-1276.

Mozharivskyj, Y., Pecharsky, A. O., Bud'ko, S. \& Miller, G. J. (2004). Chem. Mater. 16, 1580-1589.

Nelson, J., Misra, S., Yang, Y., Jackson, A., Liu, Y., Wang, H., Dai, H., Andrews, J. C., Cui, Y. \& Toney, M. F. (2012). J. Am. Chem. Soc. 134, 6337-6343.

Nylén, J., Lidin, S., Andersson, M., Iversen, B. B., Newman, N. \& Häussermann, U. (2007). Chem. Mater. 19, 834-838.

Pedersen, B. L., Birkedal, H., Frederiksen, P. T. \& Iversen, B. B. (2006). Proc. 25th Inter. Conf. Thermoelectr. pp. 520-523.

Pedersen, B. L. \& Iversen, B. B. (2008). Appl. Phys. Lett. 92, 161907.

Pedersen, B. L., Nishibori, E., Birkedal, H., Bentien, A., Sakata, M., Nygren, M., Frederiksen, P. T. \& Iversen, B. B. (2007). Chem. Mater. 19, 6304-6311.

Pedersen, B. L., Yin, H., Birkedal, H., Nygren, M. \& Iversen, B. B. (2010). Chem. Mater. 22, 2375-2383.

Pei, Y., LaLonde, A., Iwanaga, S. \& Snyder, G. J. (2011). Energy Environ. Sci. 4, 2085-2089.

Petrícek, V., Dušek, M. \& Palatinus, L. (2014). Z. Kristallogr. 229, 345-352.

Prescher, C. \& Prakapenka, V. B. (2015). High. Press. Res. 35, $223-$ 230.

Reardon, H., Blichfeld, A. B., Kasai, H., Yin, H., Bøjesen, E. D. \& Iversen, B. B. (2017). Phys. Chem. Chem. Phys. 19, 15734-15744.

Rodríguez-Carvajal, J. (1993). Phys. B Phys. Condens. Matter. 192, $55-69$.

Sadia, Y., Aminov, Z., Mogilyansky, D. \& Gelbstein, Y. (2016). Intermetallics, 68, 71-77.

Senyshyn, A., Mühlbauer, M. J., Nikolowski, K., Pirling, T. \& Ehrenberg, H. (2012). J. Power Sources, 203, 126-129.

Shaver, P. J. \& Blair, J. (1966). Phys. Rev. 141, 649-663.

Snyder, G. J., Christensen, M., Nishibori, E., Caillat, T. \& Iversen, B. B. (2004). Nat. Mater. 3, 458-463.

Taminato, S., Yonemura, M., Shiotani, S., Kamiyama, T., Torii, S., Nagao, M., Ishikawa, Y., Mori, K., Fukunaga, T., Onodera, Y., Naka, T., Morishima, M., Ukyo, Y., Adipranoto, D. S., Arai, H., Uchimoto, Y., Ogumi, Z., Suzuki, K., Hirayama M., Kanno R. (2016). Sci. Rep. 6, 28843.

Toberer, E. S., Rauwel, P., Gariel, S., Taftø, J. \& Snyder, G. J. (2010). J. Mater. Chem. 20, 9877-9885.

Topsøe, H. (2003). J. Catal. 216, 155-164.

Ulvestad, A., Singer, A., Clark, J. N., Cho, H. M., Kim, J. W., Harder, R., Maser, J., Meng, Y. S. \& Shpyrko, O. G. (2015). Science, 348, 1344-1348.

Yin, H., Blichfeld, A. B., Christensen, M. \& Iversen, B. B. (2014). ACS Appl. Mater. Interfaces. 6, 10542-10548.

Yin, H., Christensen, M., Lock, N. \& Iversen, B. B. (2012). Appl. Phys. Lett. 101, 043901.

Yin, H., Christensen, M., Pedersen, B. L., Nishibori, E., Aoyagi, S. \& Iversen, B. B. (2010). J. Elec. Mater. 39, 1957-1959. 